\title{
Láser en ortodoncia
}

\section{Laser in orthodontics}

María Ruíz-Esculpi ${ }^{1, a}$, Estela Ricse-Chaupis ${ }^{2, a}$, Judith Villanueva-Vega ${ }^{3, a}$, Liz Torres-Maita ${ }^{4, b}$.

\section{RESUMEN}

La primera aplicación del láser en un diente fue realizada en 1965. Desde entonces ha presentado una constante evolución y desarrollo. La tecnología láser permite realizar procedimientos en tejidos duros y blandos, pudiendo ser utilizado con las siguientes finalidades: como prevención de la desmineralización, en la adhesión y remoción de brackets, en la reducción del dolor producto del movimiento dental, en la reparación ósea después de la expansión, en diversas cirugías y otras aplicaciones más. El objetivo de este artículo es realizar una revisión bibliográfica sobre algunos de los usos, ventajas y características del láser en Ortodoncia.

\section{Palabras Clave: LÁSER, ORTODONCIA, ODONTOLOGÍA, ANALGESIA.}

\begin{abstract}
The first application of laser in a tooth was made in 1965. Since then this tecnology had a constant evolution and development. Laser technology allows procedures in hard and soft tissues, it can be used for the following purposes: prevention of demineralization, bonding and debonding of brackets, to reduce pain resulting from tooth movement, bone repair after expansion, surgeries and other applications. The aim of this article is to review the literature on some of the uses, advantages and features of the laser in orthodontics.
\end{abstract}

Key Words: LASER, ORTHODONTICS, DENTISTRY, ANALGESIA

Universidad Central de Venezuela. Caracas, Venezuela.

Universidad de San Martín de Porres. Lima, Perú.

Universidad Nacional Mayor de San Marcos. Lima, Perú.

Facultad de Estomatología, Universidad Peruana Cayetano Heredia. Lima, Perú.

Residente del Programa de Especialización en Ortodoncia.

b Especialista en ortodoncia. 


\section{INTRODUCCIÓN}

La utilización del láser en Odontología ha experimentado una constante evolución y desarrollo, siendo cada vez más las especialidades odontológicas que lo emplean ya sea en procedimientos diagnósticos o terapeúticos. Los láseres contemporáneos permiten llevar a cabo procedimientos en tejido duro y blando haciendo que la experiencia del dolor sea menos desagradable para los pacientes. Sin embargo existe poca información al respecto. El objetivo de esta revisión bibliográfica es conocer los usos y ventajas de la laserterapia en Ortodoncia así como sus lineamientos generales.

La palabra 'Láser' es un acrónimo de “amplificación de luz por emisión estimulada de radiación". Su base teórica fue postulada por Albert Einstein en 1916 (1). Fue Theodore H. Maiman en 1960 quien anunciara oficialmente el funcionamiento del primer láser. Un láser de rubí desarrollado en los laboratorios de investigación de la firma Hughes Aircraft Company en EE.UU (1,2). La primera aplicación del láser en un diente "in vivo" fue realizada por el dermatólogo Goldman en 1965, utilizando el láser de rubí en un diente de su hermano odontólogo, relatando tras el acto operatorio que el paciente no había sentido dolor, durante ni después de la irradiación con el láser (3).

La luz láser difiere de otras formas de luz debido a tres características: alta monocromaticidad (luz láser compuesta de fotones del mismo color), elevada coherencia (longitudes de onda viajan ordenadamente en relación al tiempo y espacio) y unidireccionalidad

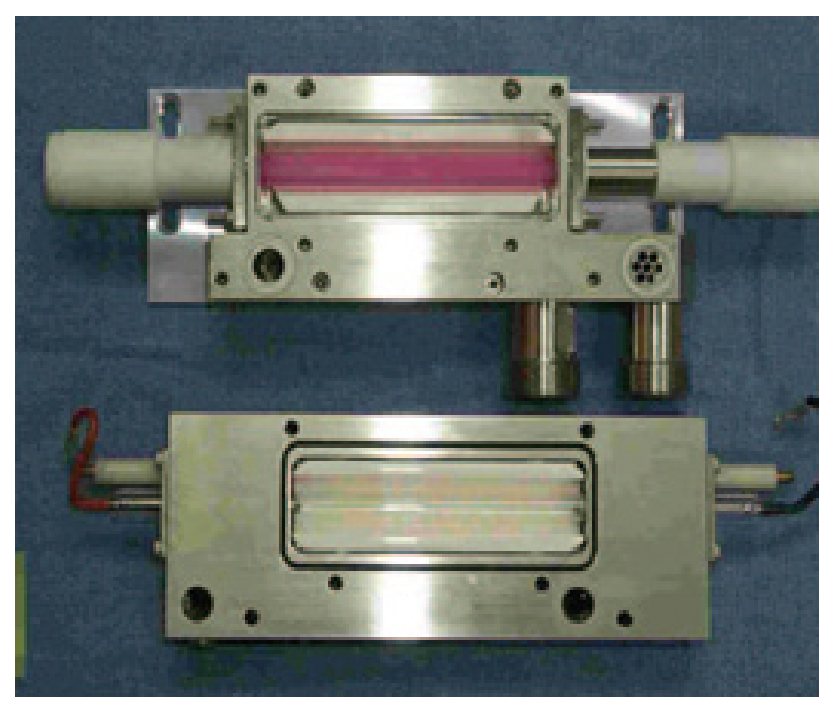

Fig 1. Láser de rubí. (luz convergente que se concentra en un punto focal). La radiación láser se encuentra en el espectro de luz que varía desde el infrarrojo a los rayos ultravioleta, pasando por el espectro visible (4). El principio del láser es generar un haz de luz monocromática (que tiene una longitud de onda muy estrecha) y altamente colimada (que tiene una sola dirección) a través de un tubo filamentoso. El tubo o fibra tiene una capa de revestimiento que colima la energía de la luz y tiene una funda protectora exterior. Esto significa que el láser se produce en la punta y no en los lados de la fibra. El láser emite energía concentrada a través de la fina punta de la fibra óptica a los tejidos donde la energía es absorbida. El grado de absorción variará con la longitud de onda del láser (medido en nanómetros), la salida de potencia o energía seleccionado por el clínico y las características ópticas del tejido objetivo incluyendo su contenido de agua (5).

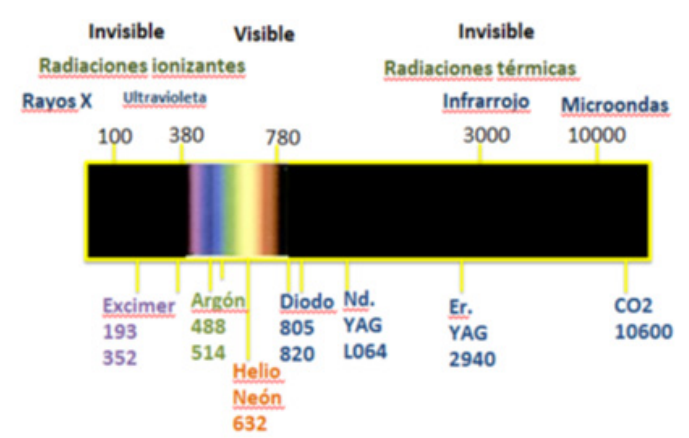

Fig 2. Espectro electromagnético.

\section{Clasificación del LÁSER}

Los láseres pueden ser clasificados de múltiples formas: en relación a su medio activo, según su longitud de onda, forma de emisión u otros criterios. La forma más habitual de clasificarlos es en razón a la potencia a la cual van a ser usados. Es así que podemos referirnos a dos grandes grupos de láseres $(6,7)$ :

- $\quad$ Láser de baja potencia.

- Láser de alta potencia.

Usos del láser en ortodoncia

El láser en Ortodoncia se puede utilizar con las siguientes finalidades: prevención de la desmineralización, adhesión, remoción de brackets, 


\begin{tabular}{|l|l|l|l|l|l|}
\hline \multicolumn{1}{|c|}{ Clasificación } & \multicolumn{1}{|c|}{ Potencia } & \multicolumn{1}{c|}{ Emisión } & \multicolumn{1}{c|}{ Efectos } & \multicolumn{1}{c|}{$\begin{array}{c}\text { Tecanismo de } \\
\text { acción }\end{array}$} \\
\hline $\begin{array}{l}\text { Láser de baja } \\
\text { potencia o tera- } \\
\text { peútico }\end{array}$ & $\begin{array}{l}\text { Menor o igual } 50 \\
\mathrm{~mW} .\end{array}$ & o Continua & $\begin{array}{l}\text { o Bioestimulante } \\
\text { o Analgésico } \\
\text { o Antiinflamatorio }\end{array}$ & $\begin{array}{l}\text { o He-Ne } \\
\text { o AsGa } \\
\text { o AsGaAl (diodo) }\end{array}$ & $\begin{array}{l}\text { Concentran menor } \\
\text { superficie de } \\
\text { aplicación mayor. }\end{array}$ \\
\hline $\begin{array}{l}\text { Láser de alta } \\
\text { potencia o quirúr- } \\
\text { gico }\end{array}$ & $\begin{array}{l}\text { Mayor o igual a } \\
\text { 0.5W. }\end{array}$ & $\begin{array}{l}\text { o Continua } \\
\text { o Pulsada }\end{array}$ & $\begin{array}{l}\text { o Efecto térmico } \\
\text { o Corte } \\
\text { o Coagulación } \\
\text { o Fotopolimerización }\end{array}$ & $\begin{array}{l}\text { o Argón } \\
\text { o Er:Yag } \\
\text { o CO2 } \text { COag }\end{array}$ & $\begin{array}{l}\text { Concentran } \\
\text { gran cantidad de } \\
\text { energía en una } \\
\text { superficie de } \\
\text { aplicación menor. }\end{array}$ \\
\hline
\end{tabular}

Cuadro 1 Clasificación del LASER

manejo del dolor, movimiento dentario, reparación ósea después de la expansión, manejo de tejidos blandos, polimerización, holografía, escáner, diagnóstico y soldadura. En esta ocasión se desarrollarán algunas de estas aplicaciones.

\section{A) Prevención de la desmineralización}

La presencia de zonas de desmineralización del esmalte clínicamente detectables después del retiro de los aparatos de ortodoncia ha sido bien identificado. Estas lesiones de mancha blanca se consideran precursoras de caries en esmalte y dentina. En ortodoncia se han atribuido a la acumulación prolongada y la retención de la placa bacteriana en la superficie del esmalte adyacente a los aparatos (8). El mecanismo más probable para la resistencia a la caries es mediante la creación de microespacios en el esmalte creados por la irradiación con láser los cuales atrapan los iones liberados y actúan como sitios de precipitación mineral. Por lo tanto, el esmalte tiene una mayor afinidad por el calcio, fosfato e iones fluoruro (9). Existen varios factores que pueden actuar en conjunto para lograr esta reducción de la susceptibilidad a la caries. Los estudios realizados indican 10 segundos de exposición a un nivel de energía de $250 \mathrm{~mW}$ mostrando una reducción de 31 $35 \%$ en la desmineralización (9). En un estudio in vitro realizado por Noel y col 9 en 86 dientes humanos se evaluó el efecto de la exposición al láser de Argón $(250 \mathrm{~mW})$ a 5 y 10 segundos en la desmineralización del esmalte; encontraron una reducción del 15 y $22 \%$ en profundidad de las lesiones a 5 y 10 segundos de exposición respectivamente en comparación con los grupos controles.

En un estudio in vivo realizado por Monseau y col 10 en nueve voluntarios cuyos planes de tratamiento incluían la exodoncia de las 4 primeras premolares, un grupo de ellos se trató con láser durante 60 segundos y otro grupo no recibió tratamiento. Se les colocó bandas a ambos grupos durante 5 semanas y luego se realizaron las extracciones. La profundidad promedio y el área promedio de la lesión en el grupo tratado con láser se redujo en un $94,1 \%$ y en $94,4 \%$ respectivamente en comparación con el grupo control. Concluyen que además de disminuir la desmineralización del esmalte y la pérdida de la estructura dental, el tratamiento con láser puede reducir el umbral del $\mathrm{pH}$ en el que se produce la disolución.

\section{B) Adhesión y remoción de brackets}

La fuerza de adhesión entre el bracket y la superficie del esmalte depende de tres factores: el mecanismo de retención de la base del bracket, el material adhesivo y la preparación de la superficie del diente. Comúnmente se utilizan sistemas adhesivos que emplean acondicionador de esmalte, solución primer, y resina para adherir los brackets de ortodoncia a la superficie del esmalte. En general, estos sistemas adhesivos contienen $35-37 \%$ de ácido ortofosfórico, lo que acondiciona la superficie del esmalte $(10,11)$. B.1) Adhesión de brackets. Los LASERES de alta intensidad Er:YAG yEr,Cr:YSGG se han utilizado para el grabado del esmalte previo al cementado de brackets. A pesar que los resultados obtenidos hasta ahora no superan los valores de adhesión que se alcanzan con el ácido ortofosfórico, puede considerarse como una técnica válida ya que superan el 70\% de retención, que es el valor mínimo requerido. Con el láser de Er:YAG el esmalte es irradiado con una longitud de onda de 2, $94 \mu \mathrm{m}, 300 \mathrm{~mJ} /$ pulso, 10 pulsos por segundo durante 10 segundos. La energía del láser permite la fusión y la ablación 
localizada de la superficie del esmalte, lo que afecta al grabado a través del proceso de vaporización y micro-explosiones continuas, que ocurren debido a la vaporización del agua atrapada dentro de la matriz de hidroxiapatita (12).Türköz y col realizaron un estudio con el objetivo de comparar el efecto de diferentes técnicas de acondicionamiento del esmalte, se utilizaron 90 premolares humanos extraídos por tratamiento ortdóntico para este estudio, los cuales fueron divididos aleatoriamente en 6 grupos de 15 especímenes cada uno. La superficie del esmalte de los dientes se grabó con $35 \%$ de ácido ortofosfórico en el Grupo 1, con un primer de auto-grabado en el Grupo 2, en el Grupo 3 se realizó un arenado, en el Grupo 4 un arenado y grabado con $35 \%$ de ácido ortofosfórico, acondicionamiento con Er: YAG en el Grupo 5 y acondicionamiento con láser Er: YAG y grabado con $35 \%$ de ácido fosfórico en gel respectivamente en el Grupo 6. Después de los procedimientos de acondicionamiento del esmalte, los brackets fueron cementados y se registró la fuerza de adhesión. Después del descementado, se calculó el índice de remanente adhesivo para todos los grupos, un diente de cada grupo fue inspeccionado por microscopio electrónico de barrido para la evaluación de las características de la superficie del esmalte. Los resultados del estudio arrojaron que el grupo con grabado ácido y uso de láser tuvo el valor más alto de fuerza de adhesión $(13.61 \pm 1.14$ $\mathrm{MPa})$ mientras que el grupo de acondicionamiento con arenado mostró el valor más bajo $(3.12 \pm 0.61$ $\mathrm{MPa}$ ). Por lo tanto, se concluyó que las técnicas de auto grabado y grabado ácido son más seguras para el cementado de brackets en ortodoncia (10).

B.2) Remoción de brackets: Para este procedimiento, se utiliza el láser de alta intensidad del grupo YAG o, en la mayoría de los casos, el láser de $\mathrm{CO} 2$ de modo pulsátil (con una longitud de onda de 10,6 micras con una potencia de 3 a $20 \mathrm{~W}$ con tiempo de exposición de 2 a 3 segundos), en el descementado de brackets cerámicos, el láser de alta potencia produce calor, lo que provoca un reblandecimiento de la resina y facilita la remoción del bracket sin que ocurra la fractura del esmalte dental o daños pulpares (4). El LASER se ha utilizado de forma experimental para despegar brackets de cerámica, donde la energía láser puede degradar la resina adhesiva como resultado de los efectos de ablandamiento térmico, la ablación térmica, y fotoablación. Se ha reportado que el aumento de la temperatura de la cavidad pulpar con la radiación láser es menor que la temperatura a la cual la pulpa dental se necrosa (5.5 C) y con el uso de una menor intensidad ayudamos a proteger la pulpa (13).

\section{C) Manejo del dolor}

En el tratamiento del dolor dentario debido a movimientos ortodónticos, el láser tiene una acción analgésica, antiinflamatoria y de reparación dentinaria. Los LÁSERES de baja potencia poseen propiedades analgésicas para disminuir el dolor tras los ajustes ortodóncicos, entre los cuales destacan el láser de Arseniuro de Galio y Aluminio (Ga,Al,As), el láser de Arseniuro de Galio (Ga,As) y el láser de HelioNeón. El láser que se utiliza en estos casos puede ser el de diodo de arseniuro de galio y aluminio, que se aplica de una manera puntual, en el ápice radicular con una dosimetría de $2 \mathrm{~J} / \mathrm{cm} 2$ y a lo largo del eje de la raíz con tres puntos de $1 \mathrm{~J} / \mathrm{cm} 2$. La frecuencia de aplicación es de 1-2 sesiones semanales $(4,14)$.

El mecanismo de acción analgésica no ha sido establecido, pero se asocia con su acción antiinflamatoria y efecto neuronal, el láser de baja frecuencia produce una estimulación benéfica de neuronas deprimidas y del sistema linfocitario, entre otras acciones neuronales se encuentra la estabilización del potencial de membrana y la liberación de neurotransmisores (14).

En un estudio de Fujiyama y col se probó la hipótesis de que no hay dolor asociado con la aplicación de fuerzas ortodónticas después de la aplicación local con la irradiación de láser de $\mathrm{CO} 2$ en los dientes involucrados. Se utilizó una muestra de 90 pacientes, a los cuales se les colocó módulos de separación a distal de los contactos en los primeros molares maxilares. En los resultados se observó una reducción significativa del dolor con el tratamiento con láser inmediatamente después de la inserción de separadores al cuarto día, pero no se observaron diferencias con el lado de control no irradiado. No se observaron diferencias significativas en la cantidad de movimiento de los dientes entre ambos grupos, con lo cual la hipótesis es rechazada. Estos resultados sugieren que la irradiación local con láser de CO2 puede reducir el dolor asociado con la aplicación de fuerzas ortodónticas sin interferir con el movimiento de los dientes (15). 
D) Efectos fotobiomoduladores del láser en ortodoncia D.1)Expansión del maxilar Los beneficios de usar el láser durante de la expansión consiste en un menor discomfort del paciente, acelerar la formación de hueso en la sutura media palatina, prevenir la recidiva de la expansión y abreviar el período de retención $(4,16,17)$. Para esta terapia se usa el láser de baja potencia Diodo de Arseniato de Gálio Aluminio por sus propiedades bioestimulantes celulares (4).

Saperiay col 18, demuestran que el nivel de ARNm de colágeno de tipo I aumentó por la irradiación con láser durante la cicatrización de heridas de la piel. Como el colágeno de tipo I es una proteína importante en la matriz ósea, la observación de la estimulación láser a nivel del colágeno es indirecta, este efecto estimulador de la irradiación con láser apoyaría la formación ósea (18). Saito y Shimitzu17, investigaron los efectos de la irradiación con láser en la regeneración ósea durante la expansión de la sutura media palatina en ratas, usando como parámetros la cantidad de hueso neoformado, área osteoide y hueso mineralizado; encontrando que la irradiación con el láser de baja potencia GaAlAs acelera la regeneración ósea, inhibe la recidiva y acorta el periodo de retención (22). Cepera y col 19, quienes usan el láser diodo durante la expansión rápida del maxilar en niños y encontraron que el láser mejora la apertura de la sutura media palatina por estimulación de la actividad osteoclástica, el cual reduce el movimiento ortodóntico indeseado, la vestibularización de las piezas dentales y acelera el proceso de regeneración ósea (19). La aplicación del láser se realiza en puntos determinados distribuidos en la trayectoria paralela a la sutura media palatina, antes de activación del expansor, con una dosis de $2 \mathrm{~J} /$ $\mathrm{cm} 2$ por punto determinado. Durante el periodo de activación de la expansión las aplicaciones deben ser realizadas de 2 a 3 sesiones semanales con intervalo mínimo de 48 horas.

D.2) Movimiento dental. Doshi y Bhad encontraron que el láser aumenta en $30 \%$ el movimiento dentario en comparación con el grupo control por lo que se concluye que la terapia con láser aumenta la velocidad de movimiento ortodóntico de los dientes de una manera fisiológica, no causa efectos secundarios sobre la vitalidad de los dientes o el periodonto. Por lo tanto, se puede utilizar de forma

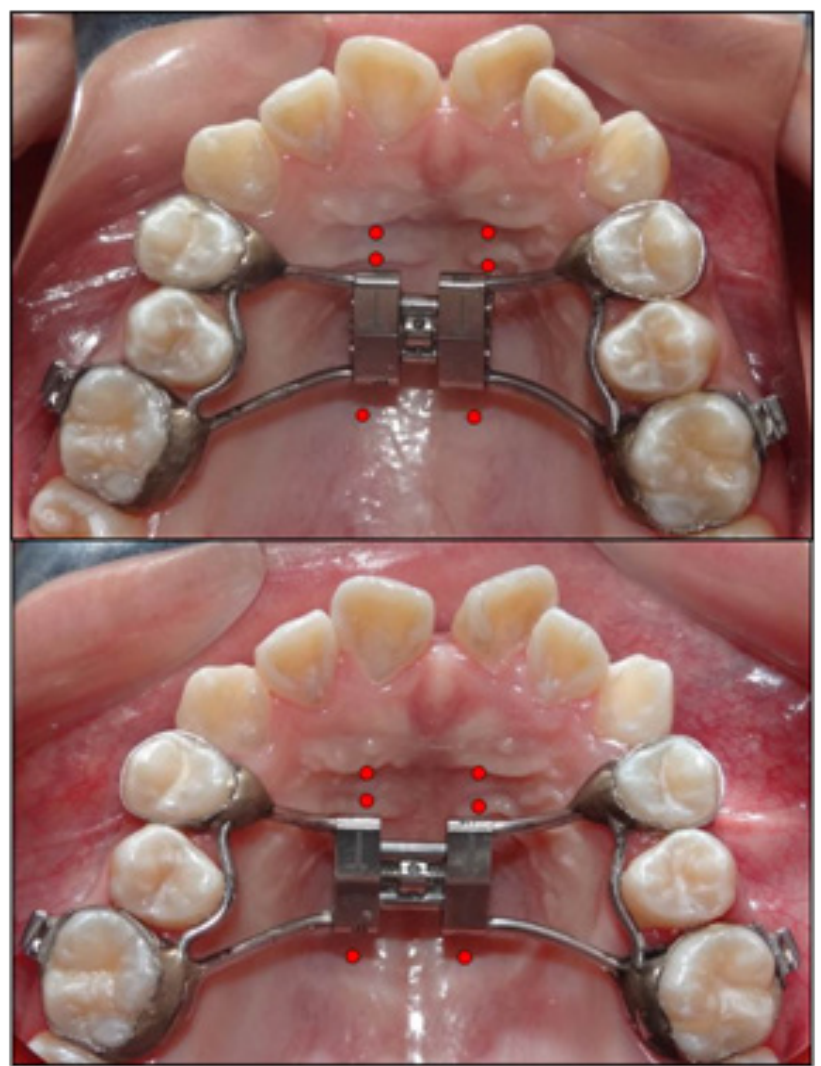

Fig 3. Puntos de aplicación del láser en la ERM.

segura y de manera rutinaria durante el tratamiento de ortodoncia para acortar el tiempo de tratamiento (18). Fujita y col 20 diseñan un estudio con el objetivo de examinar los efectos de la irradiación con láser de baja potencia (GaAlAs) en la expresión del RANK, RANKL y OPG durante el movimiento dental experimental in vivo, y en la expresión del RANK en las células precursoras de los osteoclastos in vitro en ratas. En el movimiento mesial de la molar se irradió el área alrededor de ésta, durante 3 minutos por punto (mesial, bucal y palatino) a través de la encía con un total de energía de $54 \mathrm{~J} / \mathrm{cm} 2$ por un periodo de 7 días.

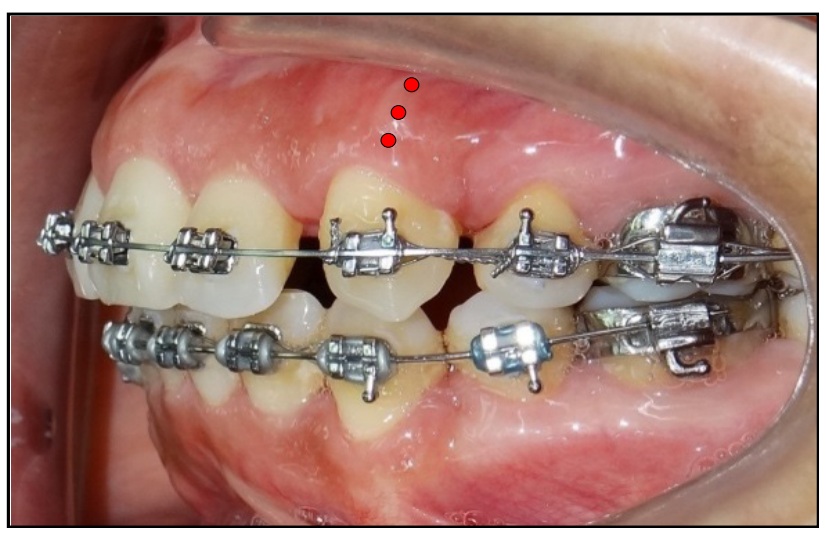

Fig 4. Puntos de aplicación del láser durante el movimiento dental. 
Se encontró que la cantidad de movimiento de los dientes fue significativamente mayor en el grupo de irradiación. La aplicación es de forma puntual, en el ápice radicular con una dosis de $2 \mathrm{~J} / \mathrm{cm} 2$ a lo largo del eje radicular con 3 puntos de aplicación de $1 \mathrm{~J} / \mathrm{cm} 2$ y la frecuencia de aplicación será de 1 a 2 sesiones semanales (4).

E) Manejo de los tejidos blandos: El láser en cirugía menor ofrece numerosas ventajas comparadas con la técnica convencional. La excisión es más precisa, coagula los vasos sanguíneos, sella los vasos linfáticos y esteriliza el campo durante la ablación, manteniendo un campo seco y limpio. Además el tiempo operatorio es menor y la recuperación postoperatoria es más rápida. La cirugía con láser se realiza rutinariamente usando solamente anestesia tópica, existe menos sangrado (particularmente en la cirugía de frenillos). En el post operatorio el paciente reporta menor discomfort y escasas complicaciones funcionales (en el habla o la masticación). Para la absorción se requiere de un absorbente de luz, denominado cromóforo, en la cavidad bucal este será la melanina, hemoglobina, y agua (21).

En situaciones graves, el paciente ortodóntico debe ser derivado al periodoncista para controlar y tratar la enfermedad periodontal presente. En estos casos, el periodoncista puede intervenir quirúrgicamente o utilizando la radiación laser, que se muestra bastante eficaz en estos procedimientos, realizando las cirugías sin dolor y con un post operatorio más cómodo para el paciente (22).

El láser de diodo es propuesto para la cirugía de los tejidos blandos debido a que las longitudes de onda de este tipo son pobremente absorbidos por la estructura

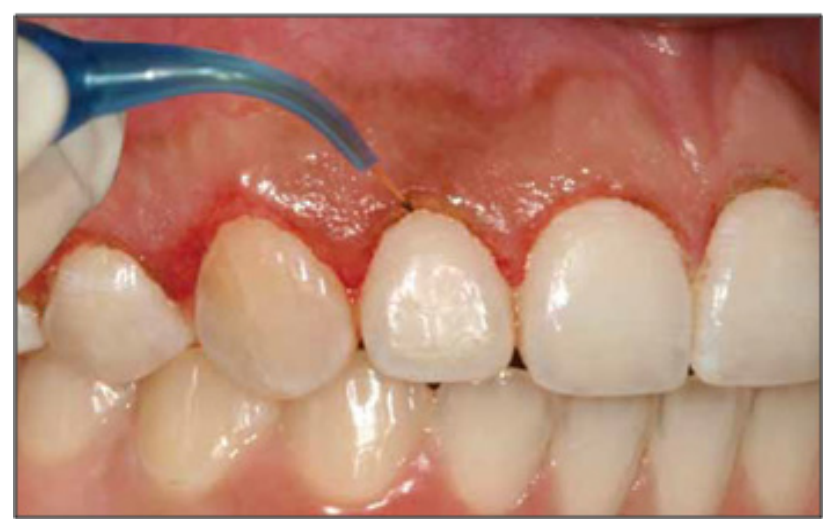

Fig 5. Aplicación del láser para la gingivectomía. dental y metales, por lo cual los procedimientos de ablación se puede realizar con seguridad en las proximidades del esmalte, aparatos de ortodoncia y dispositivos de anclaje temporales. El láser también puede ser usado en la fibrotomía y su ventaja sería un procedimiento no invasivo, según sugieren Kim y col 23. Quienes investigaron la eficacia y los efectos secundarios del láser en la fibrotomía periodontal circunferencial supracrestal en dientes rotados en beagles. Se utilizó un láser de diodo (Ga-Al-As) con una longitud de onda de $808 \mathrm{~nm}$ y el diámetro de fibra de $0,4 \mathrm{~mm}$. La punta de láser se inserta en el surco gingival con el nivel de la cresta del hueso alveolar, y la incisión se extiende alrededor de la circunferencia del diente con el sistema configurado para el modo de corte de tejidos blandos (onda continua; 1,2 W). La cantidad de recidiva, la profundidad del surco, y la recesión gingival se midieron a las 4 y 8 semanas. Se concluyó que el láser disminuye la recaída después de la rotación del diente, sin causar daño aparente a las estructuras periodontales de soporte. Además, el efecto bactericida transferido por el láser dentro de la bolsa periodontal puede reducir el riesgo de infección (23).

Olivi y col 32 evaluaron clínicamente la eficacia de Er,Cr:YSGG a una potencia de 1,5W o menos en 156 frenectomías. Se reportó una alta aceptación por el paciente y no hubieron eventos postoperatorios adversos (24). La frenectomía con láser diodo sin anestesia infiltrativa fue sugerido por Kafas y col 33, llegaron a la conclusión de que este procedimiento tiene una óptima curación post-quirúrgicamente. Sin embargo, en casos graves se puede necesitar anestesia infiltrativa. Kara 34 comparó los efectos del láser Nd:YAG y la técnica convencional en el grado de los niveles de ansiedad preoperatoria, el

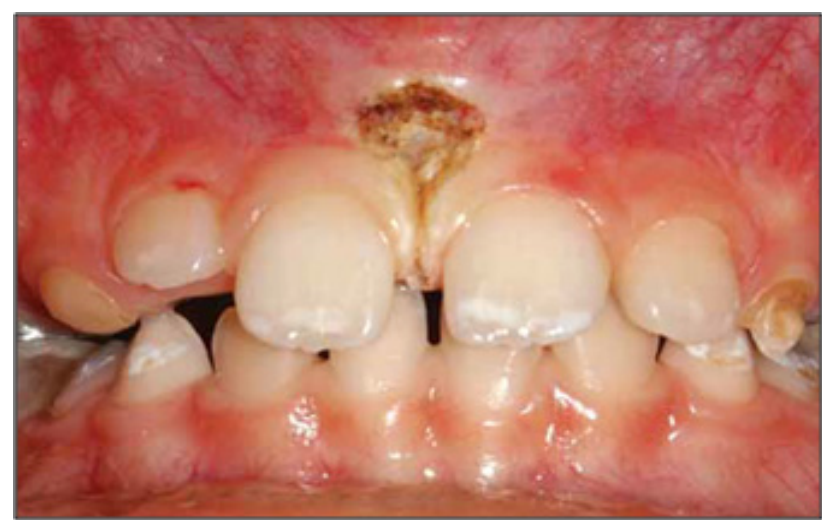

Fig 6. Frenectomía con láser. 
dolor postoperatorio, molestias y complicaciones funcionales (alimentación y habla) de la frenectomía. Los resultados sugieren que el láser Nd: YAG en el tratamiento de los tejidos blandos proporciona una mejor percepción en el paciente (25).

\section{CONCLUSIONES}

El Ortodoncista debe tener una comprensión adecuada de los principios y fundamentos de la luz láser y sus múltiples aplicaciones clínicas.

Los láseres de baja potencia son excelentes estimulantes celulares, por lo que, sus usos más comunes son: el control del dolor, el movimiento dental y complementariamente durante la expansión rápida maxilar.

Los láseres de alta potencia son una alternativa eficaz al método convencional en la adhesión y remoción de brackets sin alterar el esmalte ni dañar la pulpa dental, así como, en la prevención de la desmineralización del esmalte y el manejo de tejidos blandos durante el tratamiento ortodóntico.

\section{CORRESPONDENCIA}

\section{Liz María Torres Maita \\ DNI 20101079}

Av. La Mar 1684, Pueblo Libre, Lima

Torresmliz@hotmail.com

\section{REFERENCIAS BIBLIOGRÁFICAS}

1. Parker S. Introduction, history of lasers and laser light production. British Dent J. 2007;202(1):21-31.

2. Maiman TH. Stimulated optical radiation in ruby. Nature. 1960;187:493-4.

3. Calzava D, Herrero F, García A, Bascones A. Aplicación del láser en patología bucal (1): Introducción y efecto sobre los tejidos. Avances en Odontoestomatología. 2001;17(1):31-40.

4. Santos L, De Souza C, Castanha J, Hermont R, Pinelli R, Janson G. A utilização do laser em Ortodontia. R Dental Press Ortodon Ortop Facial 2005;10(5):149-56.

5. Sarver D, Yanosky M. Principles of cosmetic dentistry in orthodontics: Part 2. Soft tissue laser technology and cosmetic gingival contouring. Am J Orthod Dentofacial Orthop. 2005; 127:85-90.

6. España A, Arnabat J, Berini L, Gay C. Aplicaciones del láser en Odontología. Revista del ilustre consejo general de colegios de Odontólogos y Estomatólogos de España 2004;9(5):497-511.

7. Oltra D, España A, Berini L, Gay C. Aplicaciones del láser de baja potencia en Odontología. Revista del ilustre consejo general de colegios de Odontólogos y Estomatólogos de España. 2004;9(5):517-24.

8. O'Really M, Featherstone J. Demineralization and remineralization around orthodontic appliances: $\mathrm{An}$ in vivo study. Am J OrthodDentofacOrthop. 1987;92:33-40.

9. Noel L, Rebellato J, Sheats R. The Effect of Argon Laser Irradiation on Demineralization resistance of Human Enamel Adjacent to Orthodontic Brackets: An In Vitro Study. Angle Orthod 2003;73:249-258.

10. Monseau A, Kao E, Gladwin M, Benli O, Ngan P. The effects of argon laser irradiation on enamel decalcification: An in vivo study. Am J Orthod Dentofacial Orthop 2002;122: 251-9.

11.Türköz Ç, Ulusoy Ç.Evaluation of different enamel conditioning techniques for orthodontic bonding. Korean J Orthod. 2012;42(1):32-8.

12.Revilla-Gutiérrez V, Aranabat-Domínguez J, EspañaTost AJ, Gay-Escoda C. Aplicaciones de los láseres de Er:YAG y de Er,Cr:YSGG en Odontología. Revista del ilustre consejo general de colegios de Odontólogos y Estomatólogos de España 2004;9(5):551-62.

13.Lee B, Hsieh T, Lee Y, Lan W, Hsu Y, Wen P, Lin C. Bond Strengths of Orthodontic Bracket After Acid-Etched, Er:YAG Laser-Irradiated and Combined Treatment on Enamel Surface. Angle Orthod. 2003;73:565-70.

14.Iijima M, Yasuda Y, Muguruma T, Mizoguchi I. Effects of $\mathrm{CO} 2$ laser debonding of a ceramic bracket on the mechanical properties of enamel. Angle Orthod. 2010;80:1029-35.

15.Holbemrg F. Uso de láserterapeutico en el control del dolor en ortodoncia. Int. J. Odontostomat 2010:4(1);43-6.

16.Fujiyama K, Murakami T, Fujii A, Kushima K, TakanoYamamoto T. Clinical Effect of CO2 Laser in Reducing Pain in Orthodontics. Angle Orthod. 2008:78(2);299-303.

17. Ozawa Y, Shimizu N, Mishima H, Kariya G, Yamaguchi M, Takiguchi H. Stimulatory effects of low-power laser irradiation on bone formation in vitro. En: Altshuler G., Blankenou R, Wigdor H. Advanced laser dentistry. Proc, SPIE 1984. Bellingham: SPIE-the international society for optical engineering 1995;281-8.

18. Saito S, Shimizu N. Stimulatory effects of low power laser irradiation on bone regeneration in mid palatal suture during expansion in the rat. Am J Orthod Dentofacial Orthop 1997;111(5):525-32.

19.Saperia D, Glassberg E, Lyons R, Abergel R, Boneux P, Castel J. Demonstration of elevated type I and type III procollagen mRNA levels in cutaneous wounds treated with helium-neon laser. Biochem Biophys Res Commun 1986;138:1123-8.

20.Cepera F, Torres F, Scanavini M, Paranhos L, Capelozza L. Effect of a low-level laser on bone regeneration after 
rapid maxillary expansion. Am J Orthod Dentofacial Orthop. 2012;141:444-50.

21.Fujita S, Yamaguchi M, Yamamoto H, Kasai K. Low energy laser stimulates tooth movement velocity via expresion of RANK and RANKL. Orthod Carniofac res 2008;11:143-155.

22.Reza F, Katayoun K, Farzaneh A, Nikoo T. Laser in orthodontics. Silvano Naretto En: Principles in Contemporany Orthodontics. INTECH.

23. To T, Rabie B, Wonk R. McGrath C. The adjunct effectiveness of diode laser gingivectomy in maintaining periodontal health during orthodontic treatment. A randomized controlled clinical trial. Angle Orthod. 2013;83:437.

24.Kim S, Paek J, Park K, Kang S, Park Y. Laser-aided circumferential supra crestalfiberotomy and low-level laser therapy effects on relapse of rotated teeth in beagles. Angle Orthod. 2010;80(2):385-90.

25.Olivi G, Chaumanet G, Genovese M, Beneduce C, Andreana S. Er,Cr:YSGG laser labial frenectomy: a clinical retrospective evaluation of 156 consecutive cases. Gen Dent. 2010;58(3):e126-33.

26.Kara C. Evaluation of patient perceptions of frenectomy: a comparison of $\mathrm{Nd}$ : YAG laser and conventional techniques. Photomed Laser Surg. 2008;26(2):147-52.

Recibido : 11-11-2012

Aceptado: 24-05-2013 